\title{
A Mixed Resonating Modes Filter with Enhanced Stopband Performance
}

\author{
Liu He, Xingjian Zhong a) and Dexin Qu \\ College of Communications Engineering, Army Engineering University of PLA, Nanjing 210007, China. \\ a) Corresponding author: zxjcd00001@126.com
}

\begin{abstract}
In this paper, a filter of order five with enhanced stopband performance using mixed mode resonators is designed. Using different resonating mode resonators is to obtain different harmonic frequencies so that harmonics of the designed filter could be suppressed while keeping the same dominant frequency unchanged. Three different resonating modes are used in the filter design, including zero-order resonating mode inspired by epsilon-near-zero waveguide. The input and output ports are also properly designed to suppress some of the unwanted harmonics, improving the stoband performance further. Simulated results show that the designed filter has good performances.
\end{abstract}

Key words: mixed mode; frequency unchanged; zero-order; unwanted harmonics.

\section{INTRODUCTION}

In modern wireless communication system, high performance bandpass filters with small size, low insertion loss, high selectivity, wide stopband are usually demanded. Many efforts have been made to find the structures which could compose filters with high performances. Among various of structures and materials, epsilon-near-zero (ENZ) waveguide has recently attracted great attention for its inherent ability to "squeeze" and tunnel electromagnetic energy passing through very tight waveguide channels [1-3]. Energy tunneling can take place in rectangle waveguide just below the cut off frequency of the waveguide, when its effective permittivity approaches to zero. When connecting the narrow ENZ waveguide to the waveguide with a large cross section, a strong uniform electric field within the narrow waveguide could be enabled. Since the propagation wavelength at the tunneling frequency or zero-order-resonance (ZOR) approaches infinity, the electromagnetic wave will propagate without a phase delay along the ENZ waveguide and can be considered as quasi-static, even at a large distance [4]. Besides ZOR frequency, the ENZ waveguide also has a number of additional higher order resonances, which strongly depend on the length of the waveguide. In analogy with the phenomena in optical materials, the higher order resonances of the ENZ waveguide are called Fabry-Pérot (FP) resonance.

ENZ waveguide can find many applications such as dielectric permittivity measurement, filter design, etc. In [5] a first-order single band filter is designed and in [6] a dual-band filter is given using ENZ waveguide. A tunable second-order filter based on ENZ waveguide is given in [7]. Itoh gives a higher order filter with filter bandwidth enhanced [8]. ENZ waveguide can also be used to analyze ENZ metamaterial super-tunneling [9].

In this paper, a fifth-order waveguide filter is given using mixed mode resonators including ZOR resonator based on ENZ waveguide, rectangular resonator, etc. All the resonators resonate at the identical dominant frequency to support pass band creation. However, their harmonic frequencies are usually not the same since their resonating mode are different. The harmonic frequencies of the resonators are analyzed, the whole filter is designed and simulated. Simulated results show that the designed filter has not only good pass band performance, but also good stopband performance since some of the harmonics are suppressed by the filter. 


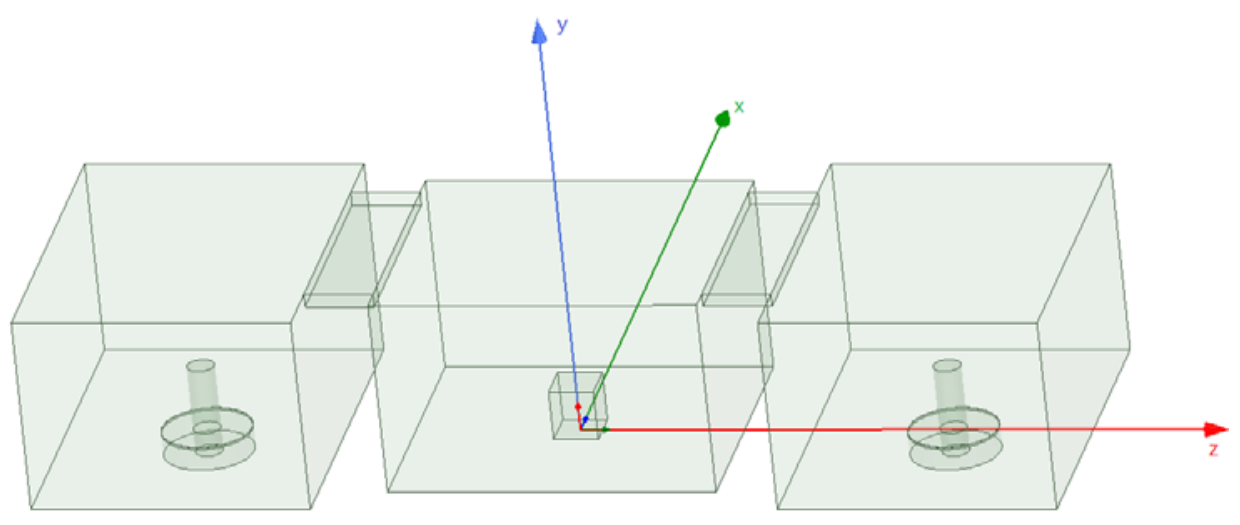

FIGURE 1. Structure of the proposed fifth-order filter

\section{ENZ WAVEGUIDE RESONATORS’ MODES}

Fig. 1 shows the simulation model of the proposed filter. The first and fifth resonators are traditional waveguide resonators directly fed with coaxial lines. The second and forth resonators are ZOR resonators constructed by ENZ waveguides with very narrow cross sections. The third resonator is constructed by a waveguide cavity loaded in the center with a metal cube.

As shown in Figure 1, when a very narrow waveguide connects to waveguides with large cross sections, it could be considered as ENZ waveguide. A proper length of this kind of ENZ waveguide can construct a ZOR resonator. The ENZ waveguide resonator with rectangular cross section could be considered as a rectangular resonator consisting of four perfect electrical conductors and two magnetic conductor walls which are perpendicular to the $\mathrm{z}$ axis. The dominant frequency is determined by the cut off frequency,

$$
f_{0}=\frac{c}{2 a \sqrt{\varepsilon_{r}}}
$$

At this frequency, the electromagnetic energy tunneling effect can be obtained in the waveguide because of the dispersive behavior of the effective permittivity in the waveguide. Also, other higher modes can be supported in the waveguide presented as harmonic frequencies. For example, letting $a=10.2 \mathrm{~mm}, b=0.5 \mathrm{~mm}$, the dominant frequency and the first two higher modes frquencies can be given out, $14.69 \mathrm{GHz}, 29.39 \mathrm{GHz}, 33.38 \mathrm{GHz}$, the electric energy distribuiton of the first two modes are given in Figure 2.

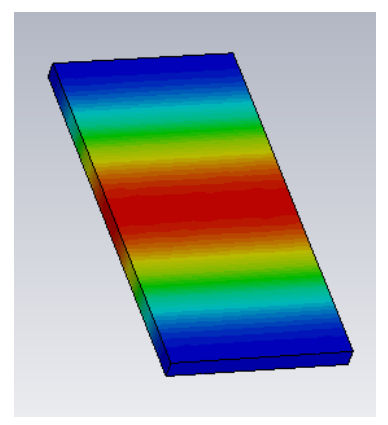

(a)

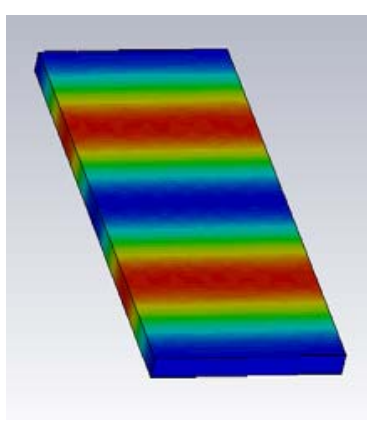

(b)

FIGURE 2. Electric field energy distribution in the waveguide. (a) dominant mode (b) the first spurious mode 
It could be easily seen that the first spurious frequency is far away from the dominant frequency. If decrease the length of the ENZ waveguide, the first spurious frequency will become larger while the dominant frequency remains unchanged. This is good for obtaining wider stopband performance.

\section{RECTANGULAR RESONATORS' MODES}

The first and fifth resonators are input and output resonators with inner conductors of the coaxial lines feeding in the center. The resonating frequency of a rectangular resonator can be calculated using formula (2),

$$
f_{m n p}=\frac{c}{2 \sqrt{\varepsilon_{r}}} \sqrt{\left(\frac{m}{a}\right)^{2}+\left(\frac{n}{b}\right)^{2}+\left(\frac{p}{l}\right)^{2}}
$$

where $\mathrm{c}$ is velocity of light in vaccum, is relative permittivity of the material filled in the cavity, a, b and 1 are dimesions of the resonating cavity in direction $\mathrm{x}, \mathrm{y}$ and $\mathrm{z}$. The subscripts $\mathrm{m}, \mathrm{n}$ and $\mathrm{p}$ are associated with resonating modes of the cavity. For example, letting $a=12.5 \mathrm{~mm}, b=16.1 \mathrm{~mm}, 1=9 \mathrm{~mm}$, the dominant frequency and the close harmonic frequencies can be given out using formula (1), $15.181 \mathrm{GHz}, 19.080 \mathrm{GHz}, 20.523 \mathrm{GHz}$. It could be clearly seen that the lowest harmonic frequency is very close to the dominant frequency. This may degrade the filter's performance. To alleviate this problem, directly coaxial feedings are used. The inner conductors of the coaxial lines are metal cylinder with radius of $0.615 \mathrm{~mm}$, height of $3.1 \mathrm{~mm}$, which is extended directly into the cavity at the center. Helped by 3D simulator, the first three resonating frequencies are given as $13.4 \mathrm{GHz}, 19.09 \mathrm{GHz}, 20.16 \mathrm{GHz}$. The distance between the second frequency and the dominant frequency is widened. The loaded rectangular resonator's mode can also easily be analyzed in 3D simulators.

\section{FILTER DESIGN}

The proposed filter is of order five, the coupling topology is shown in Figure 3,

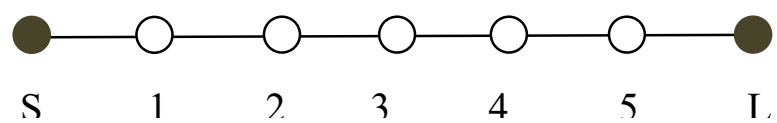

FIGURE 3. Coupling topology of the proposed filter

Where "O" denotes the ports, "○" denotes the resonators, and "—" denotes the coupling between the resonators or the resonator and the port, the associated normalized coupling matrix is shown in (3),

$$
[m]=\left[\begin{array}{ccccccc}
0 & 1.01 & 0 & 0 & 0 & 0 & 0 \\
1.01 & 0 & 0.86 & 0 & 0 & 0 & 0 \\
0 & 0.86 & 0 & 0.64 & 0 & 0 & 0 \\
0 & 0 & 0.64 & 0 & 0.64 & 0 & 0 \\
0 & 0 & 0 & 0.64 & 0 & 0.86 & 0 \\
0 & 0 & 0 & 0 & 0.86 & 0 & 1.01 \\
0 & 0 & 0 & 0 & 0 & 1.01 & 0
\end{array}\right]
$$

Based on the resonators, the filter is modeled in 3D simulator. Due to the effects of $\mathrm{I} / \mathrm{O}$ ports and couplings between resonators, the initial modeled filter's performance is not as good as expected. Through properly tuning, the filter is finally determined. Fig. 4 shows the simulated results. 


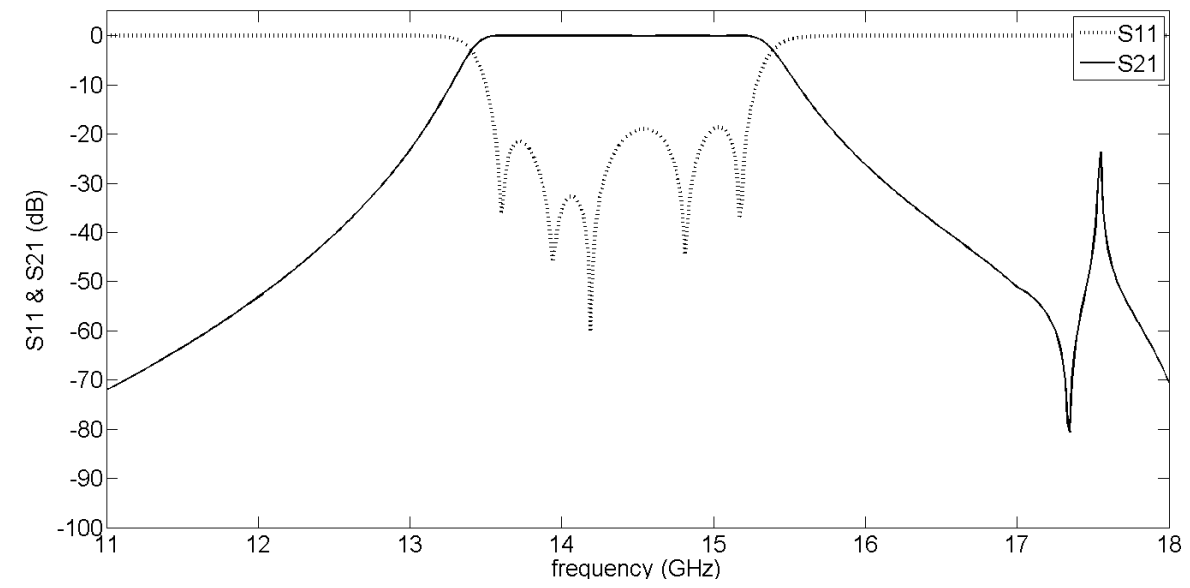

FIGURE 4. Simulated results of the designed filter

Five poles could be clearly seen in pass band in Fig. 4, showing that all the resonators work well. And in the pass band, return loss is almost larger than $20 \mathrm{~dB}$, enable good pass band performances. A spurious frequency at $17.7 \mathrm{GHz}$ appears above the pass. However, it is suppressed larger than $20 \mathrm{~dB}$ since the ENZ resonators' higher mode frequencies are far away from pass band.

\section{SUMMARY}

A waveguide filter is designed in this paper with mixed resonating modes including rectangular waveguide resonator, ZOR resonator constructed by ENZ waveguide, etc. Since resonating modes are different, the resonators usually have different spurious frequencies though they are designed with identical dominant frequency. This helps the filter widening its stopband. The input and output also properly designed with direct coaxial feedings so that some of harmonic frequencies could be suppressed by the feeding pins. The filter is optimized in $3 \mathrm{D}$ simulator. The Simulated results given show that the filter not only has good passband performance, but also good stopband performance, validating the design method in this paper.

\section{ACKNOWLEDGMENTS}

This work was supported by the National Natural Science Foundation of China (NSFC) under Grant no. 61271105 .

\section{REFERENCES}

1. M. G. Silveirinha, N. Engheta, "Theory of supercoupling, squeezing wave energy, and field confinement in narrow channels and tight bends using $\varepsilon$ near zero metamaterials," Phys. Rev. B, 76, 245109 (2007).

2. B. Edwards, A. Al'u, M. E. Young, M. Silveirinha, N. Engheta, "Experimental verification of epsilon-nearzero metamaterial coupling and energy squeezing using a microwave waveguide," Phys. Rev. Lett., 100, 033903 (2008).

3. M. Radovanociv and B. Jokanovic, "Dual-Band Filter Inspired by ENZ Waveguide", IEEE Microwave and Wireless Components Letters, 27 (6), 554-556 (2017).

4. A. Alù, M. G. Silveirinha, A. Salandrino, and N. Engheta, "Epsilon-near-zero metamaterials and electromagnetic sources: Tailoring the radiation phase pattern," Phys. Rev. B, Condens. Matter, 75(15), 14181428 (2007).

5. A. Corona-Chavez, D. V. B. Murthy, and J. L. Olvera-Cervantes, "Novel microwave filters based on Epsilon Near Zero waveguide tunnels," Microw. Opt. Technol. Lett., 53 (8), 1706-1710 (2011).

6. M. Mitrovic, B. Jokanovic, and N. Vojnovic, "Wideband tuning of the tunneling frequency in a narrowed epsilon-near-zero channel," IEEE Antennas Wireless Propag. Lett., 12, 631-634 (2013). 
7. N. Vojnovic, B. Jokanovic, M. Radovanovic, and F. Mesa, "Tunable second-order bandpass filter based on dual ENZ waveguide,” in Proc. 9th Int. Congr. Adv. Electromagn. Mater. Microw. Opt. (METAMATERIALS), Oxford, U.K., 316-318 (2015).

8. A. Corona-Chavez and T. Itoh, "Bandwidth enhancement of substrate integrated waveguide tunnels by longitudinal resonances,” in IEEE MTT-S Int. Microw. Symp. Dig. 872-875 (2010).

9. J. Bai, S. Shi, and D. W. Prather, "Analysis of epsilon-near-zero metamaterial super-tunneling using cascaded ultra-narrow waveguide channels," Prog. Electromagn. Res. M, 14, 113-121 (2010). 